\title{
Reply to 'Are atrial human pluripotent stem cell-derived cardiomyocytes ready to identify drugs that beat atrial fibrillation?'
}

\author{
Assad Shiti ${ }^{1}$, Idit Goldfracht (10 1, Naim Shaheen ${ }^{1}$, Stephanie Protze ${ }^{2}$ \& Lior Gepstein (iD) 1,3凶 \\ REPLYING to Christ et al. Nature Communications https://doi.org/10.1038/s41467-021-21949-z (2021)
}

ln n our recent report $^{1}$, we combined developmental biologyinspired differentiation strategies of human pluripotent stem cells (hPSCs) to derive chamber-specific cardiomyocytes ${ }^{2}$ and a collagen-hydrogel-based tissue engineering strategy ${ }^{3}$ to generate ring-shaped ventricular and atrial-specific engineered heart tissues (EHTs). Detailed molecular, ultrastructural, and functional phenotyping, together with targeted pharmacology, confirmed the chamber-specific identity of the atrial/ventricular EHTs, and demonstrated the potential of these models for disease modeling and drug testing applications. The latter included the ability to induce reentrant arrhythmias in the atrial EHTs and the ability to terminate such arrhythmias with established anti-arrhythmic agents (flecainide and vernakalant). In the accompanying comment, Christ et al. ${ }^{4}$ raise concerns with regards to the relative immature properties of the chamber-specific EHTs and their different response to some of the anti-arrhythmic drugs tested (vernakalant and lidocaine) in comparison to their reported effects in adult human atrial and ventricular heart tissues.

The first point raised by Christ $^{4}$ relates to vernakalant, a multichannel blocker (that also blocks the atrial-selective ionic currents $I_{\mathrm{Kur}}$ and $I_{\mathrm{KAch}}$ ), which is approved in the EU for acute conversion of atrial fibrillation $(\mathrm{AF})^{5}$. In Goldfracht et al. ${ }^{1}$, we noted significant prolongation of $\mathrm{APD}_{90}$ values following vernakalant administration to atrial EHTs. Christ et al. ${ }^{4}$ refer to two studies by Wettwer et al., ${ }^{6,7}$, in which atrial trabecula/myocytes isolated from patients undergoing open-heart surgery were studied. In one study, they noted that vernakalant administration did not lead to $\mathrm{APD}_{90}$ prolongation in isolated atrial trabecula ${ }^{6}$. In their second study ${ }^{7}$, they suggest that this lack of APD prolongation stems from the inability of $I_{\text {Kur }}$ blockade to prolong $\mathrm{APD}_{90}$ due to indirect activation of $I_{\mathrm{Kr}}$.

To address the aforementioned comment, we first aimed to reproduce vernakalant's APD-prolonging effects in a different hPSC line and using a different experimental model. To this end, we evaluated the effects of vernakalant in a two-dimensional human-induced pluripotent stem cell (hiPSC)-derived atrial cardiomyocyte cell sheet model ${ }^{8,9}$. As shown in Fig. 1, vernakalant also significantly prolonged APD values in this 2D hiPSCbased atrial tissue model. These results were further corroborated in the very recent publication of Gunawan et al. ${ }^{10}$.

We also noted in Wettwer et al. ${ }^{6}$ that although vernakalant did not alter APD in atrial trabecula, this finding was limited to patients with sinus-rhythm. In patients with chronic AF, however, vernakalant significantly prolonged $\mathrm{APD}_{90}{ }^{6}$. This finding also correlated with the second Wettwer paper, where pharmacological blockade of $I_{\text {Kur }}$ with either 4-aminopyridine or AVE0118 shortened APD in atrial cells from sinus-rhythm patients, but prolonged APD in AF patients ${ }^{7}$.

Interestingly, as described in our study ${ }^{1}$, in contrast to the ventricular EHTs, which displayed a normal activation pattern ("sinus-rhythm-like"), the vast majority of atrial EHTs displayed continuous fast and irregular arrhythmogenic activity due to multiple reentrant circuits ("AF-like") ${ }^{1}$. This arrhythmogenic activity persisted for weeks but could be terminated, at least temporarily, by electrical cardioversion to allow the drug studies. Hence, one may consider the state of the atrial EHTs more analogous to the atrial cells obtained from the AF, rather than the sinus-rhythm, patients in the Wettwer study ${ }^{6}$. Consequentially, vernakalant's APD-prolonging effects in the atrial EHTs might be in agreement with the human heart tissue studies ${ }^{6,7}$. These results may also open the road to potentially use this model to study certain aspects of atrial remodeling associated with atrial tachyarrhythmias.

The mechanisms underlying vernakalant-induced APD prolongation in the atrial EHT model was not studied. One option may be the known $I_{\text {Kur }}$ blocking effect of vernakalant, which as described in Wettwer et al. ${ }^{7}$ can also prolong APD in AF patients. Christ et al. ${ }^{4}$ insightfully raised additional possible mechanisms, including the blockade of a potential basal activity of the $I_{\text {KAch }}$ current or the potential for a reduced repolarization reserve in the

\footnotetext{
${ }^{1}$ Sohnis Research Laboratory for Cardiac Electrophysiology and Regenerative Medicine, the Rappaport Faculty of Medicine and Research Institute, TechnionIsrael Institute of Technology, Haifa, Israel. ${ }^{2}$ McEwen Center for Regenerative Medicine, University Health Network, Toronto, ON, Canada. ${ }^{3}$ Cardiology Department, Rambam Health Care Campus, Haifa, Israel.凶email: mdlior@technion.ac.il
} 
A

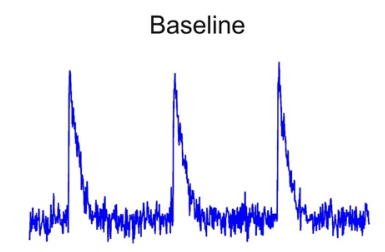

$30 \mu \mathrm{M}$ vernakalant

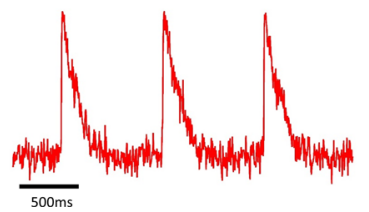

B

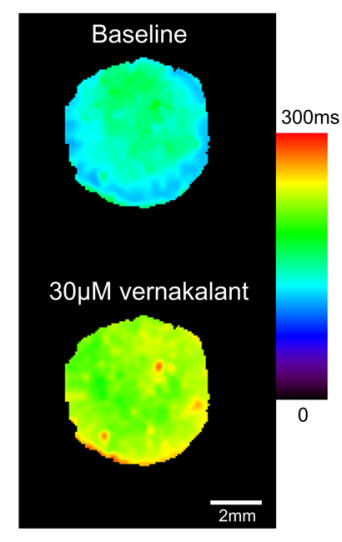

C

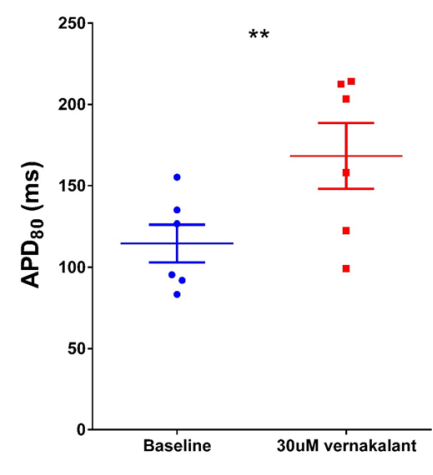

Fig. 1 Effect of vernakalant on APD in hiPSC-derived atrial cell sheets. A Example of optical action potentials acquired from the two-dimensional hiPSCderived atrial cell sheets at baseline (top) and following treatment with $30 \mu \mathrm{M}$ vernakalant (bottom). Notice the atrial-like triangular-shaped optical action potential morphologies and the longer APD after the drug treatment. B APD 80 color-coded maps depicting APD 80 values at each pixel of the atrial cell sheets at baseline (top) and following vernakalant treatment (bottom). C Summary of mean $A \mathrm{PD}_{80}$ values at baseline and upon administration of $30 \mu \mathrm{M}$ vernakalant. Note the significant ( ${ }^{\star \star} P=0.0074$, two-sided paired $t$ test, $n=6$ ) $A P D_{80}$ prolongation (from $115 \pm 12$ to $168 \pm 20 \mathrm{ms)}$ following the drug treatment. Source data is provided as a Source data file.

hPSC-derived atrial cells, as described for hPSC-derived ventricular cells ${ }^{11}$.

In our study, vernakalant was able to terminate arrhythmias in atrial EHTs and prevent their immediate reappearance. These results are in line with multiple clinical studies showing vernakalant's efficacy in terminating $\mathrm{AF}^{5}$. We did not further evaluate the mechanisms underlying vernakalant's success in terminating arrhythmias in the atrial EHT model. The APD-prolonging effect (through increase in refractory period) may contribute to this therapeutic effect, but additional contributing factors, such as vernakalant known ability to induce a frequency- and voltagedependent $I_{\mathrm{Na}}$ block may also play a significant role, since it preferably effects atrial conduction at fast rates.

The second point raised relates to vernakalant's effects on ventricular repolarization. Vernakalant-induced changes in $\mathrm{APD}_{90}$ values in the ventricular EHTs were small ( $10 \%$ rise from 403 to $445 \mathrm{~ms}$ ) relative to the large increase ( 93\%) in the atrial EHTs. Nevertheless, based on the suggestion made by Christ, we reanalyzed our data, using a pairwise statistical comparison, and noted that this small increase was statistically significant. Whether this small change, which correlates with the $\sim 20 \mathrm{~ms}$ QT interval prolongation reported in clinical studies ${ }^{5}$, is relevant to the safety of vernakalant is not known. It should be mentioned that the FDA's recent rejection to approve vernakalant use was not related specifically to QT prolongation, but rather to other adverse events (hypotension, reduced contractility, bradycardia, conduction abnormalities, etc.) and the inability to predict patients at risk ${ }^{12}$.

The third point raised by $\mathrm{Christ}^{4}$ relates to the effect of the class Ib anti-arrhythmic agent lidocaine on the chamber-specific EHTs. Lidocaine application slowed conduction in both the ventricular (by $~ 66 \%$ ) and atrial EHTs (by $~ 25 \%$ ). However, the study in the atrial EHTs was probably not powered enough to identify statistical differences. It is possible that increasing the number of experiments (beyond the current five data points that are not normally distributed) would have resulted in the statistical significance. Consequentially, we agree with $\mathrm{Christ}^{4}$ that the absence of lidocaine-induced conduction slowing should not be used as a signature of an atrial tissue phenotype. Nevertheless, it is interesting to note that the greater conduction slowing observed in the ventricular EHTs correlates with the clinical use of lidocaine in treating ventricular, but not atrial, arrhythmias.
Finally, we agree with the general notion that the relative immaturity of hPSC-derived cardiomyocytes (hPSC-CMs) in terms of their molecular, ultrastructural, metabolic, contractile, and electrophysiological properties remains a major challenge in the field. In this respect, it is important to note that the relatively immature cellular electrophysiological properties referred to in our study (depolarized RMP, slow AP upstroke) were measured from single-cell, early-stage, chamber-specific hPSC-CMs prior to their use for creation of the EHTs. It is possible that both the prolonged culture time, as well as the $3 \mathrm{D}$ engineered tissue environment and mechanical conditioning can induce a certain degree of maturation, as previously described ${ }^{13}$. Although patchclamp recordings were not repeated after EHT creation, indirect evidence, such as the response to $\mathrm{Na}^{+}$channel blockers and the somewhat improved conduction properties, may point to such a process. Ongoing efforts in the field are geared toward the development of strategies to induce the maturation status of hPSC-CMs by using combinational hormonal treatments ${ }^{14,15}$, optimizing extracellular matrix composition ${ }^{16}$, mechanical and electrical training ${ }^{13}$, and inclusion of non-myocytes cardiac cells, such as fibroblasts and endothelial cells ${ }^{17}$. Such strategies can be used in the future to also promote the maturation the chamberspecific cardiomyocytes.

In conclusion, we thank Christ et al. ${ }^{4}$ for raising these issues that helped us clarify some of the results of our study and raised awareness for the need for critical interpretation of the new model. Like any new model, the recently described chamberspecific EHTs models ${ }^{1,18,19}$ possess advantages and shortcomings, which need to be recognized for optimal model utilization and accurate interpretation of the results.

Importantly, the chamber-specific EHTs represents an unprecedented opportunity to perform high-throughput drug screens to identify potential new treatment drugs for $\mathrm{AF}$, which are eminently needed. These candidate drugs can then be further validated using adult human cardiac tissue, as suggested by Christ et $\mathrm{al}^{4}$. We and others will continue to improve the hiPSC-based cardiac tissue models, for example, by advancing its maturation status ${ }^{13,18,19}$, by introducing chamber-specific anatomical features (using organ decellularization or 3D bio-printing), and by including supporting non-myocytes ${ }^{17}$, such as cardiac fibroblasts and vascular cells to develop more clinically relevant multicellular tissue models. Introducing fibroblasts, for example, may allow to 
evaluate the role of cardiomyocyte-fibroblast interactions and, if stimulated, also of fibrosis in AF. We hope that the current work along with the discussion raised by the commentary of Christ et al. ${ }^{4}$, will advance the field forward, raise new questions and challenges, and facilitate further progress.

\section{Methods}

Generation and mapping of hiPSC-derived atrial tissues. A hiPSC-derived atrial cardiomyocyte cell sheet model was prepared, as previously described ${ }^{8,9}$. Briefly, differentiated hiPSC atrial cells were seeded as dense $20 \mu \mathrm{l}$ droplets containing $\sim 700,000$ cells on Matrigel-coated $35 \mathrm{~mm}$ plastic dishes. The resulting atrial cell sheets were cultured in $2 \mathrm{ml} \mathrm{RPMI/B27} \mathrm{medium.} \mathrm{At} \mathrm{days} \mathrm{5-10,} \mathrm{specimens} \mathrm{were}$ loaded with the voltage-sensitive dye FlouVolt and studied using an EM-CCDbased optical mapping system.

Statistical analysis. GraphPad Prism6 was used for statistical comparisons. Continuous variables are expressed as mean \pm SEM. Paired $t$ test was used to compare the effects of Vernakalant on the hiPSC-derived atrial cell sheets (Fig. 1) and on the ventricular EHTs (reanalysis of the data in Goldfract et al. ${ }^{1}$ ). $P<0.05$ was considered statistically significant.

Reporting summary. Further information on research design is available in the Nature Research Reporting Summary linked to this article.

\section{Data availability}

Source data are provided with this paper.

\section{Code availability}

Data are collected and analyzed using a custom-designed software (kindly provided by Prof. Bum-Rak Choi, Brown University).

Received: 15 June 2020; Accepted: 4 February 2021;

Published online: 19 March 2021

\section{References}

1. Goldfracht, I. et al. Generating ring-shaped engineered heart tissues from ventricular and atrial human pluripotent stem cell-derived cardiomyocytes. Nat. Commun. 11, 75 (2020).

2. Lee, J. H., Protze, S. I., Laksman, Z., Backx, P. H. \& Keller, G. M. Human pluripotent stem cell-derived atrial and ventricular cardiomyocytes develop from distinct mesoderm populations. Cell Stem Cell 21, 179-194 e174 (2017).

3. Zimmermann, W. H. et al. Tissue engineering of a differentiated cardiac muscle construct. Circ. Res. 90, 223-230 (2002).

4. Christ, T., Lemoine, M. D. \& Eschenhagen, T. Are atrial human pluripotent stem cell-derived cardiomyocytes ready to identify drugs that beat atrial fibrillation? Nat. Commun. https://doi.org/10.1038/s41467-021-21949-z (2021).

5. Roy, D. et al. Vernakalant hydrochloride for rapid conversion of atrial fibrillation: a phase 3, randomized, placebo-controlled trial. Circulation 117, 1518-1525 (2008).

6. Wettwer, E. et al. The new antiarrhythmic drug vernakalant: ex vivo study of human atrial tissue from sinus rhythm and chronic atrial fibrillation. Cardiovasc Res. 98, 145-154 (2013).

7. Wettwer, E. et al. Role of IKur in controlling action potential shape and contractility in the human atrium: influence of chronic atrial fibrillation. Circulation 110, 2299-2306 (2004).

8. Laksman, Z. et al. Modeling atrial fibrillation using human embryonic stem cell-derived atrial tissue. Sci. Rep. 7, 5268 (2017).

9. Shaheen, N. et al. Human induced pluripotent stem cell-derived cardiac cell sheets expressing genetically encoded voltage indicator for pharmacological and arrhythmia studies. Stem Cell Rep. 10, 1879-1894 (2018).

10. Gunawan, M. G. et al. Drug screening platform using human induced pluripotent stem cell-derived atrial cardiomyocytes and optical mapping. Stem Cells Transl. Med. 10, 68-82 (2020).
11. Lemoine, M. D. et al. Human induced pluripotent stem cell-derived engineered heart tissue as a sensitive test system for QT prolongation and arrhythmic triggers. Circ. Arrhythm. Electrophysiol. 11, e006035 (2018).

12. Grienberger, C. \& Konnerth, A. Imaging calcium in neurons. Neuron $\mathbf{7 3}$, 862-885 (2012).

13. Ronaldson-Bouchard, K. et al. Advanced maturation of human cardiac tissue grown from pluripotent stem cells. Nature 556, 239-243 (2018)

14. Birket, M. J. et al. Contractile defect caused by mutation in MYBPC3 revealed under conditions optimized for human PSC-cardiomyocyte function. Cell Rep. 13, 733-745 (2015).

15. Ballan, N., Shaheen, N., Keller, G. M. \& Gepstein, L. Single-cell mechanical analysis of human pluripotent stem cell-derived cardiomyocytes for drug testing and pathophysiological studies. Stem Cell Rep. 15, 587-596 (2020).

16. Herron, T. J. et al. Extracellular matrix-mediated maturation of human pluripotent stem cell-derived cardiac monolayer structure and electrophysiological function. Circ. Arrhythm. Electrophysiol. 9, e003638 (2016).

17. Giacomelli, E. et al. Human-iPSC-derived cardiac stromal cells enhance maturation in 3D cardiac microtissues and reveal non-cardiomyocyte contributions to heart disease. Cell Stem Cell 26, 862-879 e811 (2020).

18. Lemme, M. et al. Atrial-like engineered heart tissue: an in vitro model of the human atrium. Stem Cell Rep. 11, 1378-1390 (2018).

19. Zhao, Y. et al. A platform for generation of chamber-specific cardiac tissues and disease modeling. Cell 176, 913-927 e918 (2019).

\section{Acknowledgements}

This study was partially funded by the European Research Council (ERC-2017-COG773181-iPS-ChOp-AF).

\section{Author contributions}

A.S. and N.S. established the 2D hPSC-derived cardiomyocyte (atrial) cell sheet model, and performed the new optical mapping studies. S.P. established the hPSC atrial and ventricular differentiation system, and provided the atrial cells for the study. I.G. reanalyzed some of the data from the original study. A.S. and L.G. wrote the paper, and N.S., I.G., and S.P. made critical revisions to the text.

\section{Competing interests}

S.P. has an active consulting agreement with BlueRock Therapeutics LP and received funding from sponsored research agreements with BlueRock Therapeutics LP. The remaining authors declare no competing interests.

\section{Additional information}

Supplementary information The online version contains supplementary material available at https://doi.org/10.1038/s41467-021-21950-6.

Correspondence and requests for materials should be addressed to L.G.

Peer review information Nature Communications thanks Igor Efimov, Timothy Kamp and Yuji Shiba for their contribution to the peer review of this work.

Reprints and permission information is available at http://www.nature.com/reprints

Publisher's note Springer Nature remains neutral with regard to jurisdictional claims in published maps and institutional affiliations.

Open Access This article is licensed under a Creative Commons Attribution 4.0 International License, which permits use, sharing, adaptation, distribution and reproduction in any medium or format, as long as you give appropriate credit to the original author(s) and the source, provide a link to the Creative Commons license, and indicate if changes were made. The images or other third party material in this article are included in the article's Creative Commons license, unless indicated otherwise in a credit line to the material. If material is not included in the article's Creative Commons license and your intended use is not permitted by statutory regulation or exceeds the permitted use, you will need to obtain permission directly from the copyright holder. To view a copy of this license, visit http://creativecommons.org/ licenses/by/4.0/

(C) The Author(s) 2021 